\title{
Comorbidité psychiatrique au cours des événements non épileptiques : étude rétrospective dans un centre de vidéo-EEG
}

\author{
K. Mondon ${ }^{1}$, B. de Toffol ${ }^{1}$, J. Praline ${ }^{1}$, C. Receveur ${ }^{2}$, P. Gaillard ${ }^{2}$, W. El Hage ${ }^{2}$, A. Autret ${ }^{1}$ \\ 1 Clinique Neurologique, Hôpital Bretonneau, CHRU, Tours. \\ 2 Clinique Psychiatrique Universitaire, CHRU, Tours.
}

Introduction. Les événements non épileptiques (ENE) regroupent un ensemble de manifestations comportementales paroxystiques qui peuvent évoquer un diagnostic de crise d'épilepsie, mais qui ne s'accompagnent pas d'anomalies concomitantes sur l'électroencéphalogramme. L'origine retenue est primitivement psychologique.

Patients et méthodes. Nous rapportons les résultats d'une étude rétrospective précisant le diagnostic des troubles mentaux portés par les psychiatres chez des patients $(n=37)$ suspects d'ENE, ayant eu un enregistrement vidéo-EEG dans notre laboratoire.

Résultats. Suivant l'axe I du DSM-IV, 70 p. 100 des patients avaient au moins un trouble mental caractérisé au moment de leur évaluation. Les diagnostics retenus étaient, par ordre décroissant de fréquence, les états de stress post-traumatique, les autres troubles anxieux, les troubles somatoformes, les troubles dissociatifs, les troubles de l'humeur.

Conclusion. La forte association des ENE avec la pathologie psychotraumatique est discutée. Les résultats de cette étude plaident en faveur d'une prise en charge systématique de la comorbidité psychiatrique.

Mots-clés : Épilepsie, Événements non épileptiques, Troubles mentaux.

\section{Psychiatric comorbidity in patients with pseudoseizures: retrospective study conducted in a video-EEG center.}

Introduction. Pseudoseizures involve a group of paroxysmal behavioral symptoms mimicking an epileptic seizure, but without modifications of cerebral electrical activity.

Patients and method. In this retrospective study focusing on mental disorders, 37 patients with pseudoseizures were recorded during video-EEG procedure.

Results. According to DSM-IV criteria, 70 percent of the patients had at least one mental disorder when evaluated. Diagnoses were, in decreasing order of frequency: post-traumatic stress disorders, other anxious disorders, somatoform disorders, dissociative disorders, and mood disorders.

Conclusion. The strong association between pseudoseizures and post-traumatic stress disorders is discussed. Our results argue in favor of a systematic evaluation of psychiatric comorbidity.

Keywords: Epilepsy, Pseudoseizures, Mental disorders 


\section{INTRODUCTION}

Les événements non épileptiques (ENE) sont définis de manière descriptive, sans présupposé étiologique, comme « un changement brutal du comportement, des perceptions, des pensées ou des sentiments d'un sujet pendant une durée limitée, qui rappelle ou qui ressemble à une crise d'épilepsie, mais sans le concomitant électrophysiologique associé à une crise épileptique » (Betts, 1997). Le développement de la vidéo-électroencéphalographie (vidéoEEG) est à l'origine d'un regain d'intérêt pour ces manifestations qui sont examinées désormais en première intention par les épileptologues. Le support vidéo autorise une description précise des symptômes permettant de porter un diagnostic positif qui ne repose plus sur les données aléatoires de l'interrogatoire rétrospectif.

La terminologie utilisée dans la littérature pour décrire les ENE est très variée, traduisant une difficulté d'approche conceptuelle (Stone et al., 2003). Scull (1997) a répertorié 15 synonymes qui désignent ces phénomènes. Certains de ces termes ont une connotation péjorative. L'analyse psychopathologique des ENE est encore aujourd'hui fondée sur des concepts historiques, aujourd'hui revisités, comme ceux d'" hystérie ». En effet, les classifications psychiatriques descriptives modernes ont abandonné le concept général de « névroses » (DSM-IV — American Psychiatric Association, 1994).

Dans ce travail, nous avons tenté de préciser la comorbidité psychiatrique des patients avec ENE en colligeant les diagnostics psychiatriques associés. Ce travail rétrospectif correspond à une analyse pragmatique et descriptive de la prise en charge diagnostique et thérapeutique des patients examinés dans notre centre. Il a pour objectif de contribuer à une meilleure évaluation prospective du problème.

\section{PATIENTS ET MÉTHODES}

Nous avons étudié de façon rétrospective l'ensemble des dossiers cliniques des patients adultes examinés entre le $1^{\text {er }}$ janvier 1994 et le 15 février 2002 en vidéo-EEG dans le laboratoire de neurophysiologie clinique du CHRU de Tours. Les indications principales de l'examen dans notre centre sont : 1) établir le diagnostic étiologique de manifestations comportementales paroxystiques ; 2) préciser le diagnostic syndromique de patients présentant une maladie épileptique quand il est incertain ; 3) réaliser le bilan de phase I préchirurgical de certaines épilepsies pharmacorésistantes.

La durée des vidéo-EEG varie en fonction des indications (en moyenne 5 jours). L'enregistrement se déroule généralement de $9 \mathrm{~h}$ à $16 \mathrm{~h}$. En dehors de cette période, les patients restent hospitalisés. L'enregistrement électroencéphalographique est effectué à partir d'électrodes de scalp disposées suivant le montage international 10-20. Le matériel d'enregistrement électroencéphalographique se compose d'un poste d'acquisition Micromed® Numérisé (fréquence d'échantillonnage : $128 \mathrm{~Hz}$; bande passante : 0,53-50 Hz). Le comportement du sujet est enregistré par un camescope couleur SVHS de marque Sony® ou JVC@, équipé de zoom et relié à un microphone. L'électrocardiogramme des patients est systématiquement enregistré. Les paramètres sont synchronisés par horloge, mais enregistrés sur des supports différents (cassettes vidéo pour les films, disques durs pour les tracés). Pendant toute la durée de l'enregistrement, le patient est surveillé par un technicien depuis une salle contiguë. Nous avons revu tous les dossiers qui mentionnaient l'existence d'ENE. Ces dossiers ont été sélectionnés à l'aide d'une recherche par mots-clés dans les comptes rendus informatisés. Pour chaque patient, nous avons exploité les comptes rendus de consultation et les comptes rendus de vidéo-EEG dont la rédaction est standardisée et comporte : les antécédents personnels et familiaux du patient, l'histoire détaillée de sa maladie, les résultats des examens d'imagerie morphologique ou fonctionnelle, l'évaluation neuropsychologique et psychiatrique, la description détaillée des crises enregistrées tant sur le plan clinique qu'électrique. Nous avons revisionné tous les enregistrements sélectionnés. 
Au décours de l'analyse des dossiers et de la relecture des enregistrements, nous avons classé les ENE en deux groupes : groupe 1 «ENE possibles », quand les données anamnestiques et cliniques (observées par un neurologue, mais non enregistrés en vidéo-EEG) concernant ces événements se distinguent clairement d'une crise d'épilepsie ; groupe 2 "ENE certains », quand au moins un ENE a pu être enregistré en vidéo-EEG excluant une origine épileptique.

Les diagnostics psychiatriques ont été établis à partir de l'analyse rétrospective des comptes rendus des consultations spécialisées figurant dans les dossiers, provenant soit d'un psychiatre hospitalier, soit des psychiatres traitants. Les informations ont été codées suivant l'axe I du DSM-IV, qui regroupe les troubles cliniques et autres affections mentales qui peuvent faire l'objet d'une intervention clinique. Au cas par cas, les différents psychiatres ont été contactés par téléphone pour compléter nos informations. Nous présentons pour chaque patient le(s) diagnostic(s) psychiatriques qui a(ont) été porté(s).

\section{RÉSULTATS}

Le nombre total de patients adultes ayant eu une vidéo-EEG dans la période étudiée est de 213. Parmi ces 213 patients, 37 (17,4 p. 100) ont présenté des ENE, enregistrés dans 26 cas ou suspectés dans 11 cas. Douze patients ont présenté des ENE isolés (patients 1 à 12) et 25 (patients 13 à 37), des ENE associés à des crises d'épilepsie (Fig. 1).

La population d'ENE $(\mathrm{n}=37)$ comprend 24 femmes et 13 hommes (sex-ratio $=1,8)$. L'âge moyen au moment de la vidéo-EEG est de 34,3 ans : 33,9 ans pour les hommes et 34,8 ans pour les femmes. Un diagnostic selon l'axe I du DSM-IV a été porté chez 26 patients (70 p. 100) (Fig. 2). Les diagnostics psychiatriques selon l'axe I du DSM-IV sont disponibles pour chaque patient dans le tableau I. Le diagnostic le plus fréquent était un trouble anxieux $(\mathrm{n}=$ 23), surtout sous la forme d'un état de stress post-traumatique (ESPT) $(\mathrm{n}=13)$ (voir les critères dans l'annexe 1), puis, par fréquence décroissante, les troubles somatoformes $(\mathrm{n}=8)$, les troubles dissociatifs $(n=7)$ et les troubles de l'humeur $(n=7)$ (Fig. 3).

\section{DISCUSSION}

L'objet de ce travail est l'étude rétrospective des diagnostics psychiatriques retenus chez les patients enregistrés en vidéo-EEG au CHRU de Tours, et présentant des ENE soit documentés et enregistrés, soit simplement suspectés cliniquement.

Les ENE apparaissent comme très fréquents parmi les patients enregistrés dans une unité de vidéo-EEG : dans cette série, 17,4 p. 100 des patients considérés comme épileptiques ou potentiellement épileptiques ont présenté des ENE. Ce taux est comparable aux données de la littérature où la prévalence des ENE oscille entre 10 et 25 p. 100 (Gates et al., 1985 ; Gates, 2000 ; Gumnit, 1993 ; Jongsma et de Boer, 1995).

Des ENE ont pu être enregistrés durant les sessions de vidéo-EEG pour 26 des 37 patients chez lesquels ils étaient suspectés, confirmant ainsi le diagnostic. Pour les 11 autres patients, le diagnostic demeure incertain, même si des éléments cliniques forts ont conduit à retenir l'origine non épileptique des phénomènes. Nous avons choisi de ne pas exclure ces 11 patients de l'analyse de la comorbidité psychiatrique dans la mesure où il nous est apparu a posteriori que la comorbidité rencontrée et ses conséquences dans l'approche thérapeutique du malade étaient les mêmes.

Les ENE peuvent être isolés (seule manifestation clinique) ou coexister avec d'authentiques crises épileptiques : 12 à 36 p. 100 des patients présentent à la fois des crises épileptiques et d'ENE selon les populations étudiées (Betts et Boden, 1992 ; Lempert et Schmidt, 1990 ; Lesser, 1985; Ramsey et al., 1993). Dans cette série, les patients avec des ENE isolés représentent 5,6 p. 100 de la population totale ayant eu une vidéo-EEG, ceux présentant les deux types de manifestations 11,8 p. 100. Soulignons qu'il est difficile de déterminer la fréquence exacte des ENE. Cela tient: 1) à la sensibilisation au problème qui conduit à 
diagnostiquer aujourd'hui des ENE qui ne l'auraient pas été il y a quelques années ; 2) à la prévalence des ENE qui dépend de la population étudiée, celle-ci étant plus élevée dans les centres réalisant des bilans de phase I que dans les centres d'évaluation pré-chirurgicale où les patients sont déjà très sélectionnés ; 3) à la difficulté du diagnostic positif : les ENE sont souvent responsables sur les enregistrements de scalp, d'artéfacts rendant l'analyse trompeuse ; 4) aux limites de la méthode : il n'est pas toujours possible d'enregistrer des crises pendant les sessions ; 5) certaines crises d'épilepsie peuvent ne pas avoir d'expression électrique enregistrable sur un électroencéphalogramme standard ; 6) l'enregistrement d'ENE n'exclut pas que le patient présente, par ailleurs, d'authentiques crises épileptiques, et réciproquement...

L'ensemble de ces biais est à l'origine d'incertitudes diagnostiques qu'il est difficile de réduire: l'augmentation du nombre d'électrodes ou l'utilisation d'électrodes profondes (sphénoïdales par exemple), en plus de compliquer une technique simple et non invasive, n'améliorerait que partiellement la valeur prédictive de la vidéo-EEG. Pour l'un des patients de cette cohorte (observation rapportée par Drapier et al., 2001), seule l'utilisation d'électrodes implantées a permis la mise en évidence de modifications de l'électrogenèse cérébrale lors des crises, réfutant ainsi l'origine non épileptique initialement évoquée.

Certains auteurs proposent des méthodes de sensibilisation de l'enregistrement vidéo-EEG : hyperventilation, stimulation lumineuse, injection de placebo... suivis de la suggestion insistante auprès du patient du risque de survenue d'une crise (Boon et Williamson, 1993). Walczak et al. (1994) ont étudié 40 patients présentant des ENE, 20 patients avec une épilepsie, et 8 présentant les deux affections. Il a pu ainsi être déclenché des crises chez $90 \mathrm{p}$. 100 des patients présentant des ENE, dont 10 p. 100 étaient sémiologiquement différentes des crises habituelles. De plus, 25 p. 100 des patients épileptiques présentaient des événements paroxystiques dans les suites de la perfusion du placebo, dont 10 p. 100 d'authentiques crises épileptiques. Pour ces raisons, nous n'utilisons pas dans notre pratique ces techniques, et c'est conscients des nombreuses limites de la vidéo-EEG que nous en interprétons les résultats. Malgré cela, elle reste actuellement la technique de référence pour le diagnostic étiologique des événements critiques (French, 1995 ; Roberts, 1998).

Parmi les patients présentant des ENE, nous avons observé une forte proportion de troubles mentaux repérables sur l'axe 1 du DSM : parmi les 29 patients ayant eu une évaluation psychiatrique au cours de leur prise en charge, 26 (70 p. 100) présentent au moins un diagnostic suivant l'axe I du DSM-IV. Parmi les diagnostics psychiatriques retenus, nous avons observé une forte proportion d'ESPT (41 p. 100 dans le groupe de patients présentant uniquement des ENE, et 32 p. 100 dans le groupe présentant à la fois des ENE et des crises épileptiques, 35 p. 100 en moyenne). Ces taux sont très supérieurs à ceux observés dans la population générale qui sont évalués entre 1 et 14 p. 100 (American Psychiatric Association, 1994). Nous pensons que, dans cette série, ces taux sont sous-estimés puisque de nombreux patients présentant des ESPT ont en fait été uniquement diagnostiqués comme ayant des troubles somatoformes ou dissociatifs fréquemment associés aux ESPT. En effet, la direction des entretiens psychiatriques était libre, et il n'était pas demandé aux praticiens de vérifier systématiquement l'existence d'un ESPT ou d'un traumatisme psychique. Par ailleurs et selon les écoles, les troubles observés dans ces populations peuvent être analysés dans des cadres nosographiques différents : par exemple, dans le DSM-IV, la dissociation est décrite comme une rupture dans les fonctions normales d'intégration de la conscience, de la mémoire, de l'identité ou de la perception de l'environnement. Cette description est donc principalement orientée vers les symptômes psychologiques.

Plusieurs auteurs avancent l'idée que les symptômes somatoformes correspondent aussi à des phénomènes dissociatifs (Kihlstrom, 1994 ; Nemiah, 1991 ; Nijenhuis et al., 1996), classés comme tels dans la classification des troubles mentaux de l'OMS (World Health 
Organization, 1990). S'il est classique d'observer une prédominance de femmes dans les populations de patients présentant des ENE (Reuber et al., 2003), il est intéressant d'observer que la répartition hommes/femmes dans cette série (environ 2 femmes pour 1 homme) correspond exactement à la répartition hommes/femmes observée chez les patients présentant un ESPT (Breslau et al., 1991 ; Kessler et al., 1995).

Longtemps considérés comme des formes de conversion hystériques (Slavney, 1994 ; Israël, 1976), alors que le cadre des névroses était démantelé dans les classifications psychiatriques modernes (DSM-IV — American Psychiatric Association, 1995 ; CIM-10 — World Health Organization, 1990), de récents concepts sont venus s'ajouter à l'approche «névrotique » des ENE. Cela pour plusieurs raisons : 1) de nombreux patients présentant des ENE confient avoir été violés (Alper et al., 1993 ; Kuyk et al., 1999 ; Abubakr et al., 2003 ; Prigatano et al., 2003) ; 2) les psychiatres spécialisés dans la pathologie psychotraumatique ont mis en évidence des altérations de la conscience chez les patients atteints de troubles dissociatifs et de personnalités multiples.

Certains auteurs considèrent les mouvements désordonnés observés dans certaines crises pseudo-épileptiques comme la reviviscence comportementale de l'opposition des patients au traumatisme qu'ils ont subi (Goodwin, 1993). Pour d'autres auteurs (Bowman, 1993), les activités motrices observées pendant les crises peuvent correspondre à des sentiments comme la rage, la peur... Cette conception peut s'étendre aux troubles somatoformes ou aux maladies dans lesquelles des crises pseudo-épileptiques peuvent aussi s'observer. Pour ces auteurs, l'origine de la dissociation psychique est bien un traumatisme réel.

Dans notre série, 68 p. 100 des patients présentant des ENE étaient également atteints d'une maladie épileptique. La comorbidité fréquente des ENE et d'une épilepsie authentique (32 p. 100 dans une récente série de plus de 1500 patients - Martin et al., 2003) est un problème intéressant et controversé. Hormis le problème de l'incertitude diagnostique, plusieurs hypothèses ont été envisagées pour expliquer cette forte association : 1) les patients épileptiques présentent souvent des réactions émotionnelles liées à leur maladie neurologique qui peuvent s'accompagner de modifications comportementales ou végétatives ressemblant à des ENE (anxiété anticipatoire par exemple) ; 2) il existe une plus forte prévalence de troubles mentaux chez les patients épileptiques, certains d'entre eux pouvant être confondus avec des ENE (Lancman et al., 1993 ; Mondon et al., 2002) ; 3) les patients épileptiques, comme tout malade atteint d'une pathologie chronique, bénéficient vis-à-vis de l'entourage social et familial de bénéfices secondaires liés à ce statut dont les ENE pourraient être le mode d'expression comportementale. Ainsi, des cas de patients guéris d'une épilepsie temporale par une chirurgie et ayant développé dans un second temps des ENE ont pu être rapportés. De la même manière, Kapur et al. (1995) ont rapporté trois observations de patients ayant présenté des crises d'allure partielle simple d'origine non épileptique durant une vidéo-EEG dans le but de pouvoir bénéficier d'une intervention chirurgicale. Certains auteurs ont émis l'hypothèse qu'il existait une relation entre des décharges épileptiques limbiques et les symptômes dissociatifs (Mesulam, 1981 ; Rosenstein, 1994 ; Schenk et Bear, 1981). Pour d'autres, il n'y a aucun rapport entre décharges épileptiques et symptômes dissociatifs (Devinsky et al., 1989 ; Lowenstein et Putnam, 1988 ; Ross et al., 1989). Pour d'autres encore, cette forte association s'explique par la présence fortuite d'un facteur psychotraumatique dans les deux populations (Alper et al., 1997).

\section{CONCLUSION}

Les ENE sont fréquemment rencontrés en pratique clinique. Cette fréquence doit conduire à envisager systématiquement ce diagnostic devant des manifestations comportementales paroxystiques (Reuber et Elger, 2003), et cela même chez des patients présentant une authentique maladie épileptique. 
À ce jour, la vidéo-EEG demeure l'outil le plus performant pour le diagnostic positif d'ENE (Iriarte et al., 2003). L'analyse de ces phénomènes doit intégrer la dimension psychopathologique tant dans l'analyse des manifestations comportementales que dans celle de la comorbidité psychiatrique associée (Harden et al., 2003). Si les ENE répondent, par définition, aux critères du DSM-IV de trouble de conversion (Annexe 2), nous observons de façon pragmatique que les diagnostics les plus souvent retenus chez les patients de notre cohorte par les psychiatres rentrent dans le cadre des pathologies psychotraumatiques (ESPT, troubles somatoformes, troubles dissociatifs). Si ces données préliminaires doivent être confirmées par des travaux méthodologiquement plus valides, cette dimension nous parait indispensable à considérer dans la prise en charge de ces patients, dont elle conditionne le pronostic (Reuber et al., 2003 ; Kanner, 2003 ; Farias et al., 2003).

\section{RÉFÉRENCES}

ABUBAKR A, KABLINGER A, CALDITO G. (2003). Psychogenic seizures: clinical features and psychological analysis. Epilepsy Behav, 4: 241-245.

ALPER K, DEVINSKY O, PERRINE K, VASQUEZ B, LUCIANO D. (1993). Non-epileptic seizures and childhood sexual and physical abuse. Neurology, 43: 1950-1953.

ALPER K, DEVINSKY O, PERRINE K, LUCIANO D, VAZQUEZ B, PACIA S, RHEE E. (1997). Dissociation in epilepsy and conversion non epileptic seizures. Epilepsia, 38: 991997.

AMERICAN PSYCHIATRIC ASSOCIATION. (1994). Manuel diagnostique et statistique des troubles mentaux, 4e édition, Washington DC, Masson Éditeur, Paris. 1008 pp.

BETTS T, BODEN S. (1992). Diagnosis, management and prognosis of a group of 128 patients with non-epileptic attack disorder. Part I. Seizure, 1:19-26.

BETTS T. (1997). Psychiatric aspects of nonepileptic seizures. In: Epilepsy: a comprehensive textbook. Edited by J Engel Jr and TA Pedley. Lippincott-Raven Publishers, Philadelphia.

BOON PA, WILLIAMSON PD. (1993). The diagnosis of pseudoseizures. Clin Neurol Neurosurg, 95: 1-8.

BOWMAN ES. (1993). Etiology and clinical course of pseudoseizures: relationship to trauma, depression and dissociation. Psychosomatics, 34: 333-342.

BRESLAU N, DAVID GC, ANDRESKI P, PETERSON E. (1991). Traumatic events and posttraumatic stress disorder in an urban population of young adults. Arch Gen Psychiatry, 48: $216-222$.

DEVINSKY O, PUTNAM F, GRAFMAN J, BROMFIELD E, THEODORE WH. (1989). Dissociative states and epilepsy. Neurology, 39: 835-840.

DRAPIER S, BIRABEN A, DE TOFFOL B, DUHURT J, SEIGNEURET E, SCARABIN JM. (2001). Crises à manifestations négatives : à propos d'un cas exploré en stéréoélectroencéphalographie. Épilepsies, 13: 91-96.

FARIAS ST, THIEMAN C, ALSAADI TM. (2003). Psychogenic nonepileptic seizures: acute change in event frequency after presentation of the diagnosis. Epilepsy Behav, 4: 424-429.

FRENCH J. (1995). Pseudoseizures in the era of video-electroencephalogram monitoring. Curr Opin Neurol, 8: 117-120.

GATES JR, RAMANI V, WHALEN S, LOEWENSON R. (1985). Ictal characteristics of pseudoseizures. Arch Neurol, 42: 1183-1187.

GATES JR. (2000). Epidemiology and classification of non-epileptic events. In: Non epileptic seizures, 2nd ed. Gates JR, Rowan AJ., editors. Boston: Butterworth-Heinemann, pp. 3-14.

GOODWIN JM. (1993). Childwood sexual abuse and non-epileptic seizures. In: Nonepileptic seizures. Rowan A, Gates J. ed. Boston: Butterworth-Heinemamn ; p. 181-191.

GUMNIT RJ. (1993). Psychogenic seizures. In: The treatment of epilepsy: principles and practice. Wyllie E. Ed. Philadelphia, Pa: Lea \& Febiger ; pp. 692-695. 
HARDEN CL, BURGUT FT, KANNER AM. (2003). The diagnostic significance of videoEEG monitoring findings on pseudoseizure patients differs between neurologists and psychiatrists. Epilepsia, 44: 453-456.

IRIARTE J, PARRA J, URRESTARAZU E, KUYK J. (2003). Controversies in the diagnosis and management of psychogenic pseudoseizures. Epilepsy Behav, 4: 354-359.

ISRAËL L. (1996). L’hystérique, le sexe et le médecin. Éditions Masson. JONGSMA MJ, DE BOER HM. (1995). Characteristics of the population of an observation unit in a special center of epilepsy. Epilepsia, 36: S16.

KANNER AM. (2003). More controversies on the treatment of psychogenic pseudoseizures: an addendum. Epilepsy Behav, 4:360-364.

KAPUR J, PILLAI A, HENRY T. (1995). Psychogenic elaboration of simple partial seizures. Epilepsia, 36: 1126-1136.

KESSLER RC, SONNEGA A, BROMET E, HUGHES M, NELSON CB. (1995). Posttraumatic stress disorder in the national comorbidity survey. Arch Gen Psychiatry, 52: 1048-1060.

KIHLSTROM JF. (1994). One hundred years of hysteria. In: Dissociation: clinical and theorical perspectives. SJ Lynn, JW Rhue (Eds) New York : Guilford Press. pp. 365-395.

KUYK J, SPINHOVEN PH, VAN DYCK R. (1999). Hypnotic recall: a positive criterion in the differential diagnosis between epileptic and pseudo-epileptic seizures. Epilepsia, 40: 485491.

LANCMAN ME, BROTHERTON TA, ASCONAPE JJ, PENRY JK. (1993). Psychogenic seizures in adults : a longitudinal analysis. Seizure, 2: 281-286.

LEMPERT T, SCHMIDT D. (1990). Natural history and outcome of psychogenic seizures: a clinical study of 50 patients. J Neurol, 237:35-38.

LESSER RP. (1985). Psychogenic seizures. In: Recent advances in epilepsy, Vol 2. Edited by Pedley TA, Meldrum BS. Edinburgh, Churchill Livingstone, pp. 273-296.

LOEWENSTEIN RJ, PUTNAM FW. (1988). A comparasion study of dissociative symptoms in patients with complex partial seizures, MDP, and post-traumatic stress disorder. Dissociation, 4: 17-23.

MARTIN R, BURNEO JG, PRASAD A, POWELL T, FAUGHT E, KNOWLTON R, MENDEZ M, KUZNIECKY R. (2003). Frequency of epilepsy in patients with psychogenic seizures monitored by video-EEG. Neurology, 61: 1791-1792.

MESULAM MM. (1981). Dissociative states with abnormal temporal lobe EEG: multiple personality and the illusion of possession. Arch Neurol, 38: 176-181.

MONDON K, SUZANNE I, DE TOFFOL B. (2002). Épilepsie et troubles mentaux : comorbidité dépressive et anxieuse. Épilepsies, 14:23-31.

NEMIAH JC. (1991). Dissociation, conversion and somatization. In: Review of psychiatry (Vol 10). A Tasman, SM Goldfinger (Eds). Washington DC, American psychiatric press. pp. 248-260.

NIJENHUIS ERS, SPINHOVEN PH, VAN DYCK R, VAN DER HART O, VANDERLINDEN J. (1996). The development and psychometric characteristics of the somatoform dissociation questionnaire (SDQ-20). J Nerv Ment Dis, 184: 688-694.

PRIGATANO GP, STONNINGTON CM, FISHER RS. (2002). Psychological factors in the genesis and management of nonepileptic seizures: clinical observations. Epilepsy Behav, 3: 343-349.

RAMSEY R, CHEN A, BROWN M. (1993). Co-existing epilepsy and non epileptic seizures. In: Non-epileptic seizures. Rowan A, Gates J ed. Boston: Butterworth-Heinemamn, pp. 47-54. REUBER M, QURISHI A, BAUER J, HELMSTAEDTER C, FERNANDEZ G, WIDMAN G, ELGER CE. (2003). Are there physical risk factors for psychogenic non-epileptic seizures in patients with epilepsy? Seizure, 12: 561-567. 
REUBER M, ELGER CE. (2003). Psychogenic nonepileptic seizures: review and update. Epilepsy Behav, 4: 205-216.

REUBER M, PUKROP R, BAUER J, HELMSTAEDTER C, TESSENDORF N, ELGER CE. (2003). Outcome in psychogenic nonepileptic seizures: 1 to 10-year follow-up in 164 patients. Ann Neurol, 53: 305-311.

ROBERTS R. (1998). Differential diagnosis of sleep disorders, nonepileptic attacks and epileptic seizures. Curr Opin Neurol, 11:135-139.

ROSENSTEIN LD. (1994). Potential neuropsychologic and neurophysiologic correlates of multiple personality disorder. Neuropsychiatry Neuropsychol Behav Neurol, 7: 215-229.

ROSS CA, HEBER S, ANDERSON G, NORTON GR, ANDERSON BA, DEL CAMPO M, PILLAY N. (1989). Differenciating multiple personality disorders and complex partial seizures. Gen Hosp Psychiatry, 11:54-58.

SCHENK L, BEAR D. (1981). Multiple personality and related dissociative phenomena in patients with temporal lobe epilepsy. Am J Psychiatry, 138: 1311-1316.

SCULL DA. (1997). Pseudoseizures or non-epileptic seizures (NES) ; 15 synonyms. J Neurol Neurosurg Psychiatry, 62: 200.

SLAVNEY PR. (1994). Pseudoseizures, sexual abuse, and hermeneutic reasoning. Comprehensive psychiatry, 35: 471-477.

STONE J, CAMPBELL K, SHARMA N, CARSON A, WARLOW CP, SHARPE M. (2003). What should we call pseudoseizures? The patient's perspective. Seizure, 12: 568-572.

WALCZAK TS, WILLIAMS DT, BERTEN W. (1994). Utility and reliability of placebo infusion in the evaluation of patients with seizures. Neurology, 44: 394-399.

WORLD HEALTH ORGANIZATION, DIVISION OF MENTAL HEALTH. (1990). The ICD-10 classification of mental and behavioral disorders: clinical descriptions and diagnostic guidelines. Geneva: World Health Organization. 